\title{
Targeted Gene Disruption of OsCERK1 Reveals Its Indispensable Role in Chitin Perception and Involvement in the Peptidoglycan Response and Immunity in Rice
}

\author{
Yusuke Kouzai, ${ }^{1}$ Susumu Mochizuki, ${ }^{1}$ Keisuke Nakajima, ${ }^{1}$ Yoshitake Desaki, ${ }^{2}$ Masahiro Hayafune, ${ }^{2}$ \\ Hideo Miyazaki, ${ }^{2}$ Naoki Yokotani, ${ }^{1}$ Kenjirou Ozawa, ${ }^{1}$ Eiichi Minami, ${ }^{1}$ Hanae Kaku, ${ }^{2}$ Naoto Shibuya, ${ }^{2}$ \\ and Yoko Nishizawa ${ }^{1}$
}

${ }^{1}$ Genetically Modified Organism Research Center, National Institute of Agrobiological Sciences, Tsukuba, Ibaraki, 305-8602 Japan; ${ }^{2}$ Department of Life Sciences, School of Agriculture, Meiji University, Tama-ku, Kawasaki, Kanagawa, 214-8571 Japan

Submitted 6 March 2014. Accepted 26 May 2014.

OSCERK1 is a rice receptor-like kinase that mediates the signal of a fungal cell wall component, chitin, by coordinating with a lysin motif (LysM)-containing protein CEBiP. To further elucidate the function of OsCERK1 in the defense response, we disrupted $O S C E R K 1$ using an Agrobacteriummediated gene targeting system based on homologous recombination. In OSCERK1-disrupted lines, the generation of hydrogen peroxide and the alteration of gene expression in response to a chitin oligomer were completely abolished. The OsCERK1-disrupted lines also showed lowered responsiveness to a bacterial cell wall component, peptidoglycan. Yeast two-hybrid analysis indicated that OsCERK1 interacts with the LysM-containing proteins LYP4 and LYP6, which are known to participate in the peptidoglycan response in rice. Observation of the infection behavior of rice blast fungus (Magnaporthe oryzae) revealed that disruption of OsCERK1 led to increased hyphal growth in leaf sheath cells. Green fluorescent protein-tagged OsCERK1 was localized around the primary infection hyphae. These results demonstrate that OSCERK1 is indispensable for chitin perception and participates in innate immunity in rice, and also mediates the peptidoglycan response. It is also suggested that OsCERK1 mediates the signaling pathways of both fungal and bacterial molecular patterns by interacting with different LysM-containing receptor-like proteins.

Plants initiate defense responses upon perceiving structurally conserved molecules, known as microbe-associated molecular patterns (MAMPs). These molecules are perceived by pattern recognition receptors (PRR) (Boller and Felix 2009; Monaghan and Zipfel 2012). The PRR that have been identified to date are the receptor-like protein kinases (RLK) and receptor-like proteins (RLP) localized in the plasma membrane (PM) (Monaghan and Zipfel 2012). Studies have shown that RLK containing leucine-rich repeat motifs in the extracellular domain perceive peptidic MAMPs. In Arabidopsis thaliana,

Corresponding author Y. Nishizawa; E-mail: ynishi@affrc.go.jp

* The $\boldsymbol{e}$-Xtra logo stands for "electronic extra" and indicates that five supplementary figures and four supplementary tables are published online.

(C) 2014 The American Phytopathological Society
FLS2 and EFR recognize the flg22 peptide derived from bacterial flagella and the elf 18 peptide from the bacterial elongation factor Tu, respectively (Gomez-Gomez and Boller 2000; Zipfel et al. 2006). The perception of carbohydrate MAMPs that contain $N$-acetylglucosamine is mediated by PRR with lysin motifs (LysM): LysM-RLK and LysM-RLP (Monaghan and Zipfel 2012).

Chitin, a $\beta$-1,4-linked polymer of $N$-acetylglucosamine, is a constituent of fungal cell walls. Its oligomers are a typical carbohydrate MAMP, and they elicit various defense responses in many plant species (Kombrink et al. 2011). In Arabidopsis, a LysM-RLK, AtCERK1, is essential for perceiving chitin elicitors $(\mathrm{CE})$ and plays a role in immunity to fungal pathogens (Miya et al. 2007; Wan et al. 2008). AtCERK1 directly binds CE and is sufficient for CE perception (Iizasa et al. 2010; Petutschnig et al. 2010; Shinya et al. 2012). In rice, CEBiP, a LysM-RLP, directly binds CE, whereas OsCERK1, a homolog of AtCERK1, does not (Kaku et al. 2006; Shinya et al. 2012). CEBiP interacts with OsCERK1 in a CE-dependent manner. Reduced expression of either CEBiP or OsCERK1 in RNA interference (RNAi) lines resulted in an impaired response to CE (Kaku et al. 2006; Shimizu et al. 2010), indicating that both of these molecules are required for chitin signaling. Therefore, the $\mathrm{CE}$ receptor system in rice is different from that in Arabidopsis, which does not require CEBiP-like molecules (Shinya et al. 2012). To further clarify the role of OsCERK1, analysis using OsCERK1-disrupted rice plants is desirable because the RNAi lines retained some responsiveness; thus, it is possible that other LysM-RLK are also involved in the response to CE. Moreover, although CEBiP was shown to participate in immunity (Kishimoto et al. 2010; Kouzai et al. 2014), the involvement of OsCERK1 has not been analyzed.

Peptidoglycan (PGN), which contains $\beta$-1,4-linked glycan chains of alternating $\mathrm{N}$-acetylglucosamine and $\mathrm{N}$-acetylmuramic acid, is a constituent of bacterial cell walls. This polymer elicits defense responses in Arabidopsis and rice (Erbs et al. 2008; Gust et al. 2007; Liu et al. 2012). Arabidopsis has three LysMRLP (LYM1 to LYM3). LYM1 and LYM3 bind PGN and participate in the PGN response (Willmann et al. 2011). AtCERK1, which itself does not bind PGN, also acts as a PGN receptor, although biochemical interaction between LYM1 or LYM3 and AtCERK1 has not been reported (Willmann et al. 2011). The homologs of LYM1 or LYM3 in rice, LYP4 and LYP6, also bind PGN. Reduced expression of LYP4 or LYP6 
led to an impaired response to PGN (Liu et al. 2012). Because LYP4 and LYP6 lack intracellular domains, they are assumed to cooperate with LysM-RLK to mediate PGN signaling in rice. However, such a molecule has not been identified.

The subcellular localization of PRR fused to fluorescent protein has been analyzed in cells treated with MAMPs or invaded by filamentous pathogens. After stimulation with flg22, FLS2-green fluorescent protein (GFP) was transferred

A
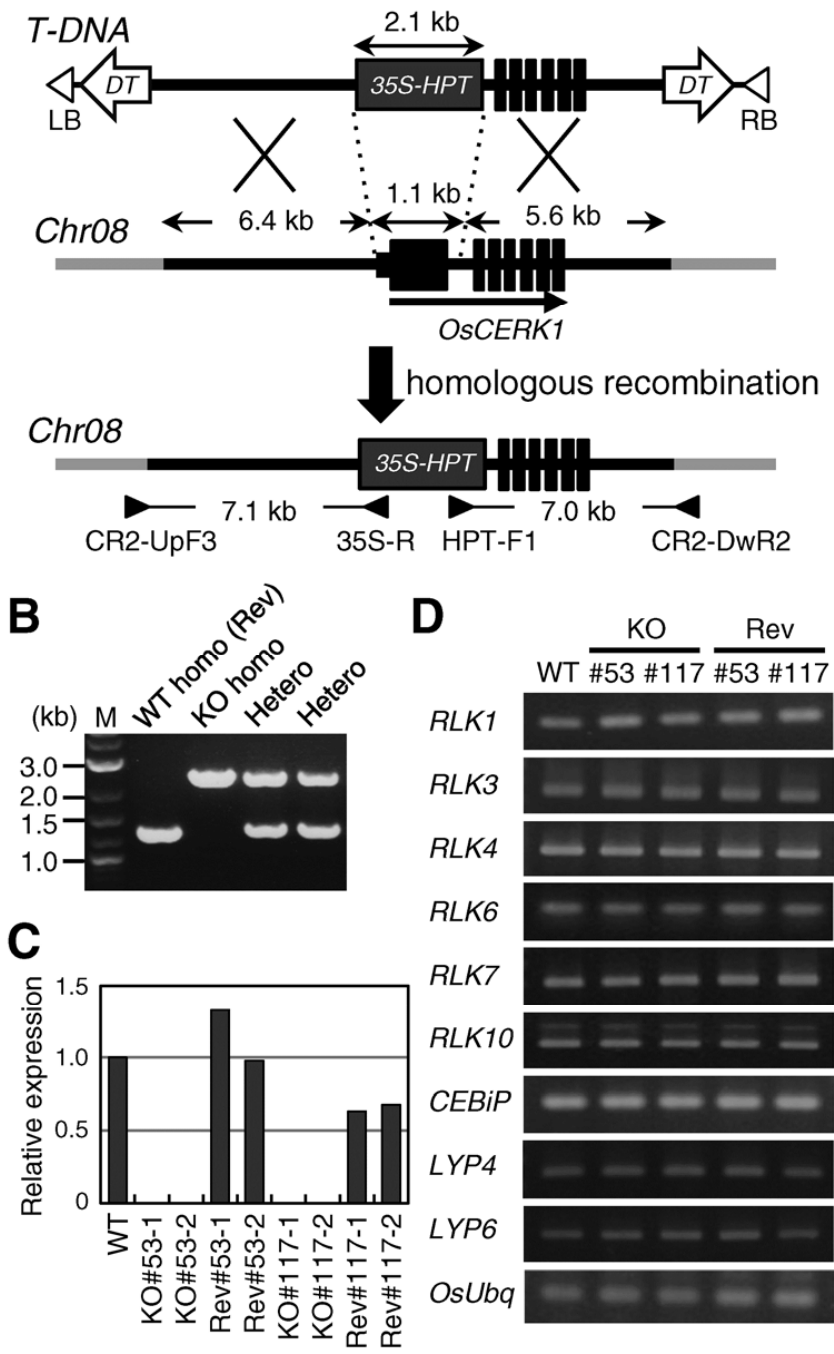

RLK6
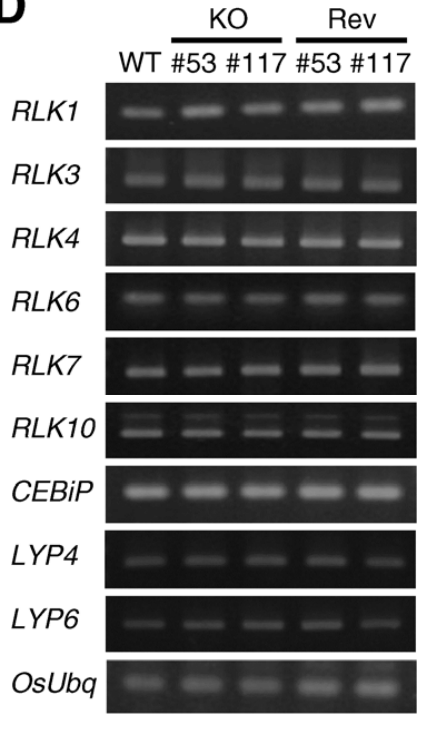

Fig. 1. Generation of OsCERK1-disrupted rice plants. A, Schematic representation of disruption of $O S C E R K 1$ by homologous recombination. Homologous recombination event via double crossovers at hygromycin phosphotransferase (HPT)-flanking homologous regions in T-DNA replaces a part of the OsCERK1 locus (Os08g0538300; $1.1 \mathrm{~kb})$ with the $H P T$ cassette $(2.1 \mathrm{~kb})$. Black arrowheads indicate position of polymerase chain reaction (PCR) primers used to screen disrupted lines. Fragments of 7.1 and $7.0 \mathrm{~kb}$ are amplified only from lines in which the expected homologous recombination occurs. $D T=$ diphtheria toxin A fragment gene, $\mathrm{LB}=$ left border of T-DNA, RB = right border of T-DNA. B, Representative result of PCR analysis to genotype progeny of genetargeted line $\left(\mathrm{T}_{1}\right.$ generation). $\mathrm{M}=\mathrm{DNA}$ molecular size marker, Rev = segregated wild-type, $\mathrm{KO}=$ knock-out. $\mathbf{C}$, Quantitative reversetranscriptase (RT)-PCR analysis of OsCERK1 expression. Transcript levels relative to that in nontransformed callus are shown. WT = nontransformed callus, $\mathrm{KO}=$ homozygous oscerk 1 mutant callus, Rev = segregated wild-type callus. D, RT-PCR analysis of expression of rice LysM-containing receptor-like protein kinase $(R L K)$ and receptor-like protein $(R L P)$ genes. WT $=$ nontransformed callus, $\mathrm{KO}=$ homozygous oscerk 1 mutant callus, Rev = segregated wild-type callus. The expression of $R L K 2,-5$, and -8 was not detected even in WT. from the PM to intracellular vesicles, and then degraded (Robatzek et al. 2006). During infection by Hyaloperonospora arabidopsidis, FLS2-GFP was located in the extrahaustorial membrane (Lu et al. 2012), a novel host cell membrane encasing the haustorium to separate the host cell from the oomycete. Transient expression of OsCERK1-GFP in rice protoplasts showed that OsCERK1 was transported from the endoplasmic reticulum to the PM by vesicle trafficking (Chen et al. 2010). However, the subcellular localization of OsCERK1 in infected rice cells remains unknown.

Although gene disruption mutants are well developed in Arabidopsis, it is still difficult to obtain such mutants in other higher plants. In rice, retrotransposon Tos 17-induced insertion mutants and T-DNA insertion lines are available (Jeon et al. 2000; Miyao et al. 2003) but do not include OsCERK1-disrupted lines. Gene disruption through homologous recombination has become a routine technique to investigate gene functions in mammals (Jasin et al. 1996). Terada and associates (2002) first reported targeted homologous recombination in the vicinity of a target gene in rice using a positive/negative (hygromycin phosphotransferase gene $[H P T] /$ diphtheria toxin A fragment gene $[D T-A]$ ) selection system. Recently, Ozawa and associates (2012) improved this technique by optimizing culture conditions, which allowed the production of $C E B i P-$ disrupted rice plants (Kouzai et al. 2014).

To elucidate the roles of OsCERK1 in the defense responses and immunity of rice, we generated OsCERK1-disrupted rice plants (oscerk1) using the Agrobacterium-mediated gene-targeting system based on homologous recombination. Here, we show that the response to chitin oligomers in rice completely depends on OsCERK1. Immunity to rice blast fungus was reduced in oscerkl, and fluorescence imaging of inoculated rice leaf sheaths revealed that GFP-tagged OsCERK1 was localized around primary infection hyphae. Furthermore, we show that OsCERK1 participates in the response to PGN, probably in cooperation with one or both LYP4 or LYP6.

\section{RESULTS}

Generation of $O s C E R K 1$-disrupted rice plants.

The binary vector used to disrupt OsCERK1 contains the $H P T$ cassette for positive selection, the upper and lower regions of the OsCERK1 first exon, and two DT-A cassettes for negative selection of random integration. Thus, the OsCERK1 first exon is replaced with the HPT cassette, following the occurrence of targeted homologous recombination (Fig. 1A). We screened 1,248 independent hygromycin-resistant calli by polymerase chain reaction (PCR) that amplified 7.1- and 7.0-kb junction fragments (Fig. 1A), and obtained five gene-targeted lines $(0.40 \%)$. Plants were regenerated from four lines, three of which showed normal growth (lines 19, 53, and 117). A representative result of genotyping of $\mathrm{T}_{1}$ plants at the OSCERK1 locus is shown in Figure 1B. A $\chi^{2}$ analysis of the segregation ratio indicated that the $H P T$ cassette in place of OsCERK1 was inherited by the $\mathrm{T}_{1}$ generation in a Mendelian manner (Supplementary Table S1).

A genomic DNA-blot analysis indicated that the first exon of OsCERK1 was deleted in the homozygous oscerk1 mutant lines, and that HPT was integrated into only the target locus (Supplementary Fig. S1A and B). DNA sequencing of the $15-\mathrm{kb}$ fragments covering the OsCERK1 locus in $\mathrm{T}_{1}$ seedlings confirmed that the gene replacement occurred at the expected sites in homozygous oscerkl plants, and that segregated hygromycin-sensitive plants had the wild-type OsCERK1 in all three lines. OsCERK1 transcripts were detected in segregated wild-type lines but not in homozygous oscerkl lines (Fig. 1C). The transcript levels of $C E B i P$ and other LysM-containing $R L K$ and $R L P$ 
genes that were expressed in cultured cells were not affected in oscerk1 or the segregated wild-type lines (Fig. 1D). These data confirmed the OsCERK1-specific disruption in the lines created.

\section{Responsiveness to CE is lost in oscerk1.}

To evaluate the effect of OsCERKI disruption on CE perception, we analyzed hydrogen peroxide $\left(\mathrm{H}_{2} \mathrm{O}_{2}\right)$ generation and gene expression profiles after $\mathrm{CE}$ treatment. When suspensioncultured cells and leaves of oscerkl were treated with $\mathrm{CE}$, they could not generate $\mathrm{H}_{2} \mathrm{O}_{2}$ (Fig. 2A and B). The ability to generate $\mathrm{H}_{2} \mathrm{O}_{2}$ in response to $\mathrm{CE}$ was recovered by introducing OsCERK1 cDNA, and $\mathrm{H}_{2} \mathrm{O}_{2}$ was generated in a kinase-activitydependent manner (Supplementary Fig. S2). Microarray analysis demonstrated that, in nontransformed cells, 1,391 genes were upregulated $(\geq \times 2)$ and 617 were downregulated $(\leq \times 1 / 2)$ after 1 h of CE treatment (Fig. 2C; Supplementary Tables S2 and S3). In oscerkl cells, none of these genes responded to CE. As a control, we confirmed that the response to a bacterial MAMP, flg22, was not affected in oscerkl (Supplementary Fig. S3).

\section{OsCERK1 contributes}

to innate immunity to Magnaporthe oryzae.

Next, we evaluated the immunity to rice blast caused by Magnaporthe oryzae in oscerkl plants. Spray inoculation of leaf blades with a compatible strain showed no significant dif- ference in lesion formation between oscerkl and nontransformed plants (data not shown). Thus, we evaluated the early infection process of an incompatible strain by scoring hyphal growth in the epidermal cells under a microscope. There was a greater extent of penetration and colonization of infection hyphae in oscerkl leaf sheaths than in wild-type ones (Fig. 3).

To analyze the cellular localization of OsCERK1 in infected cells, we produced transgenic rice plants expressing OsCERK1GFP and observed GFP fluorescence in the leaf sheaths. In uninoculated tissues, OsCERK1-GFP was present in the PM, the endoplasmic reticulum-like structures, and vesicle-like organelles (Supplementary Fig. S4). Five independent transgenic lines showed similar fluorescence patterns. As shown in Figure 4 , in the cells invaded by infection hyphae of a compatible strain (Ina86-137), GFP signals were detected around the primary infection hyphae as well as in the PM at $30 \mathrm{~h}$ postinoculation (hpi). When the infection hyphae penetrated into neighboring rice cells (42 hpi), the GFP signals disappeared from the first-invaded cell, which suggests that the rice cell was dead. In the neighboring cells, GFP signals were present again around the invading hyphae and in the PM.

Involvement of OsCERK1 in the peptidoglycan response.

Next, we examined the participation of OsCERK1 in the response to PGN. $\mathrm{H}_{2} \mathrm{O}_{2}$ generation after treatment with PGN
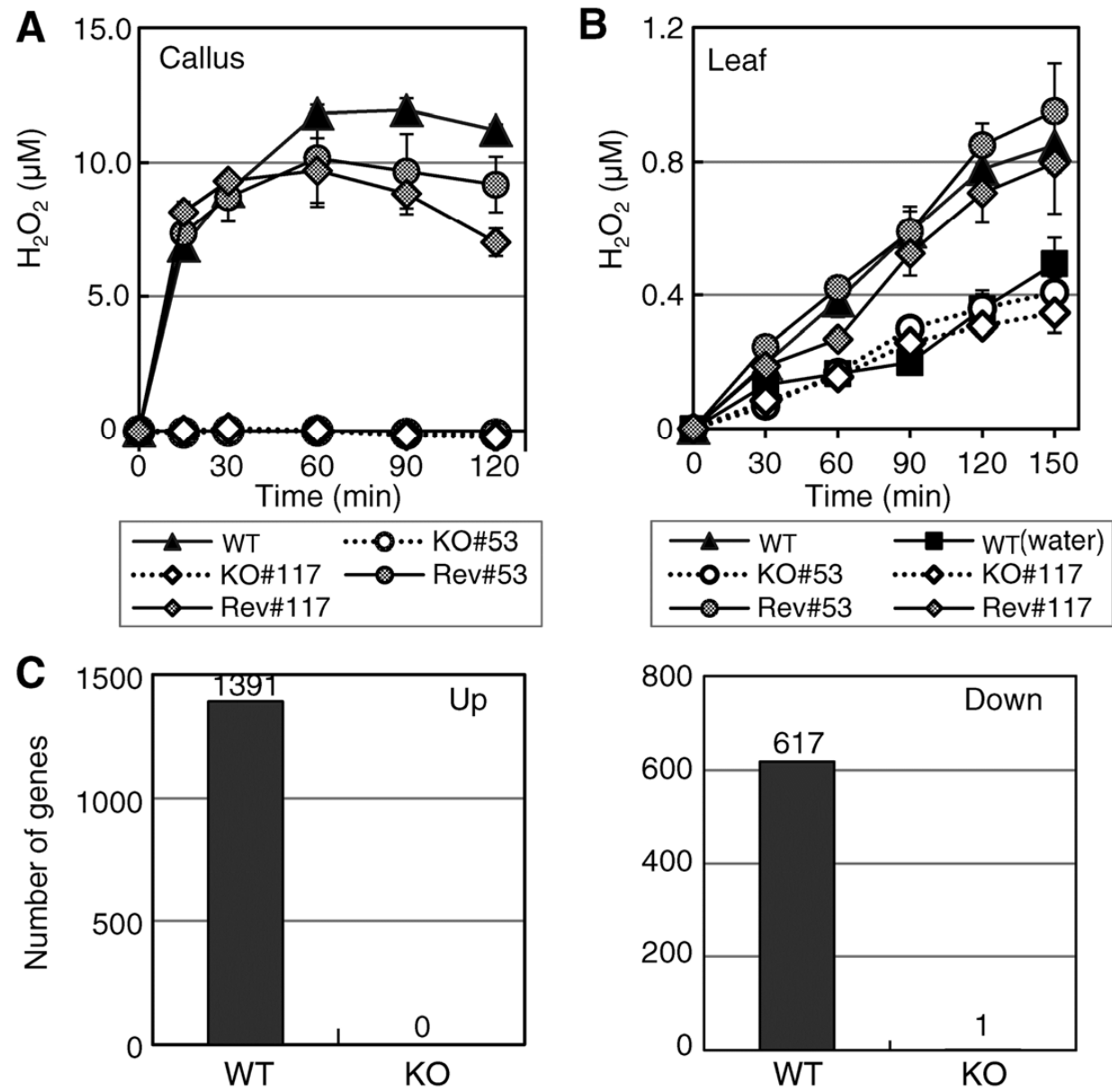

Fig. 2. Responses to chitin elicitor (CE). A, Generation of $\mathrm{H}_{2} \mathrm{O}_{2}$ in response to $\mathrm{CE}$ in cell lines. Suspension-cultured cells ( $100 \mathrm{mg}$ ) were treated with $N$-acetylchitoheptaose at $100 \mathrm{ng} \mathrm{ml}^{-1}$, and $\mathrm{H}_{2} \mathrm{O}_{2}$ in the culture solution was quantified. WT = nontransformant, $\mathrm{KO}=$ two independent homozygous oscerkl (lines 53 and 117), $\mathrm{Rev}=$ segregated wild-type lines. $\mathrm{B}$, Generation of $\mathrm{H}_{2} \mathrm{O}_{2}$ in response to CE in leaves. Sliced leaf blades (20 $\mathrm{mg}$ ) floating in water were treated with $\mathrm{N}$-acetylchitoheptaose at $100 \mathrm{ng} \mathrm{ml}^{-1}$, and $\mathrm{H}_{2} \mathrm{O}_{2}$ in the culture solution was quantified. WT $=$ nontransformant, WT (water) $=$ mock-treatment, $\mathrm{KO}=$ homozygous oscerk $1, \mathrm{Rev}=$ segregated wild-type plants. Error bars in A and B represent standard deviation $(n=3$ independent treatments). $\mathbf{C}$, Changes in gene transcript levels in response to CE. Total RNAs were extracted from suspension-cultured cells treated with $N$-acetylchitoheptaose at $100 \mathrm{ng} \mathrm{ml}^{-1}$ for $1 \mathrm{~h}$ and used for microarray analysis. Bars show number of CE-responsive genes. Up $=$ fold changes $\geq \times 2$, Down $=\leq \times 1 / 2$, WT $=$ nontransformed cells, KO $=$ oscerkl cells (line 53). Only one gene was downregulated specifically in oscerk1. 
from Bacillus subtilis or Staphylococcus aureus was partially impaired in the oscerkl cell line (Fig. 5A; Supplementary Fig. S5A). Pretreatment of PGN with lysozyme to hydrolyze the glycan chains negated the effect of OsCERK1 disruption (Fig. $5 \mathrm{~B})$, further indicating the involvement of OsCERK1 in the PGN response. A yeast two-hybrid analysis demonstrated that OsCERK1 interacted with LYP4 and LYP6, as well as with CEBiP, suggesting that OsCERK1 may play a role in perceiving PGN along with one or both LYP4 and LYP6 (Fig. 5C).

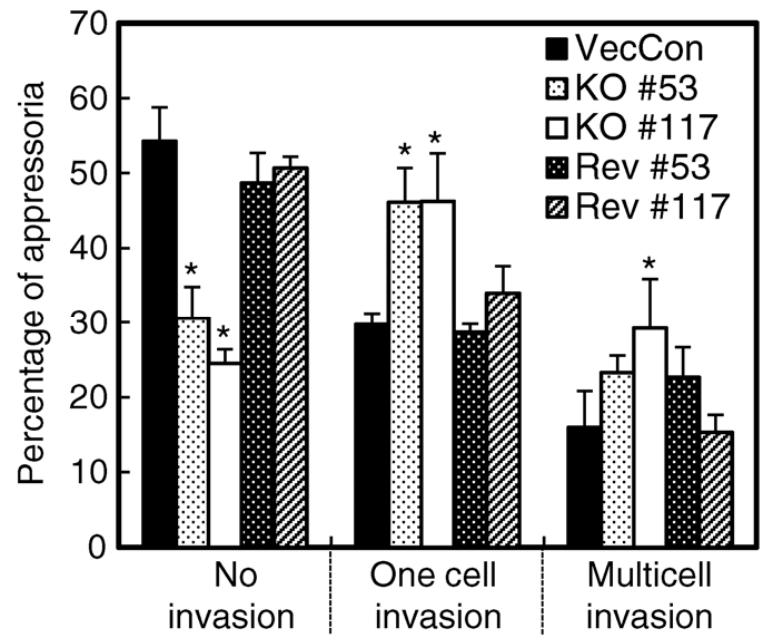

Fig. 3. Effect of OsCERK1 disruption on immunity to Magnaporthe oryzae. Sixth leaf sheaths of two independent oscerk1 lines were inoculated with $M$. oryzae (Guy11, an incompatible strain). Degrees of invasion were scored for 100 appressoria per plant under a microscope at $48 \mathrm{~h}$ postinoculation and are shown as percentages of total numbers of appressoria in three categories. $\mathrm{KO}=$ homozygous oscerk 1 , Rev = segregated wild-type plants. Error bars represent standard deviation $(n=4$ sibling plants in the $\mathrm{T}_{2}$ generation). Asterisks indicate significant differences compared with the vector control (VecCon) at $P<0.05$ (Dunnett's test). Experiments were performed twice; representative data are shown.
To examine the effect of OsCERK1 disruption on immunity to a bacterial pathogen, we inoculated oscerkl leaves with Xanthomonas oryzae pv. oryzae, the causal agent of rice bacterial leaf blight. We measured lesion area at 14 days postinoculation (dpi) but OsCERK1 disruption had no detectable effect (Fig. 6A). We also evaluated the bacterial population at $4 \mathrm{dpi}$ using wild-type $X$. oryzae pv. oryzae or the $X$. oryzae pv. oryzae hrpX mutant, which lacks the ability to deliver type III effectors and, thus, pathogenicity, and obtained the same results (Fig. 6B and C).

\section{DISCUSSION}

In OsCERK1-RNAi cells, the responses to CE were not completely abolished (Shimizu et al. 2010). Therefore, in this study, we first aimed to clarify whether the remaining responsiveness was because of other LysM or low levels of OsCERK1 transcripts. We generated three independent oscerkl lines using an Agrobacterium-mediated gene targeting system based on homologous recombination (Ozawa et al. 2012). The frequency of double crossovers at the OsCERK1 locus was $0.40 \%$ in positive/negative selected calli, which was similar to that for the $C E B i P$ locus (0.47\%; Kouzai et al. 2014). The disruption of OsCERK1 did not cause gamete or embryo lethality. Using oscerk 1 and OsCERK1-complemented lines, we demonstrated that OsCERK1 with kinase activity is indispensable for the response to $\mathrm{CE}$ in rice (Fig. 2). Thus, the contribution of OsCERK1 to CE perception is the same as that of AtCERK1 in Arabidopsis (Miya et al. 2007), although OsCERK1 needs CEBiP in contrast to AtCERK1, which is an "all-in-one" receptor (Shinya et al. 2012). Recently, using a CEBiP-disrupted mutant, we showed that CEBiP is the major CE-binding protein in rice and almost all of the CE responsiveness in cultured cells is attributable to CEBiP (Kouzai et al. 2014). The results of that study and the present study revealed the mechanism of CE perception in rice; almost all CE signals are recognized by $\mathrm{CEBiP}$ and every $\mathrm{CE}$ signal is transmitted into cells via OsCERK1.

\section{$30 \mathrm{hpi}$}
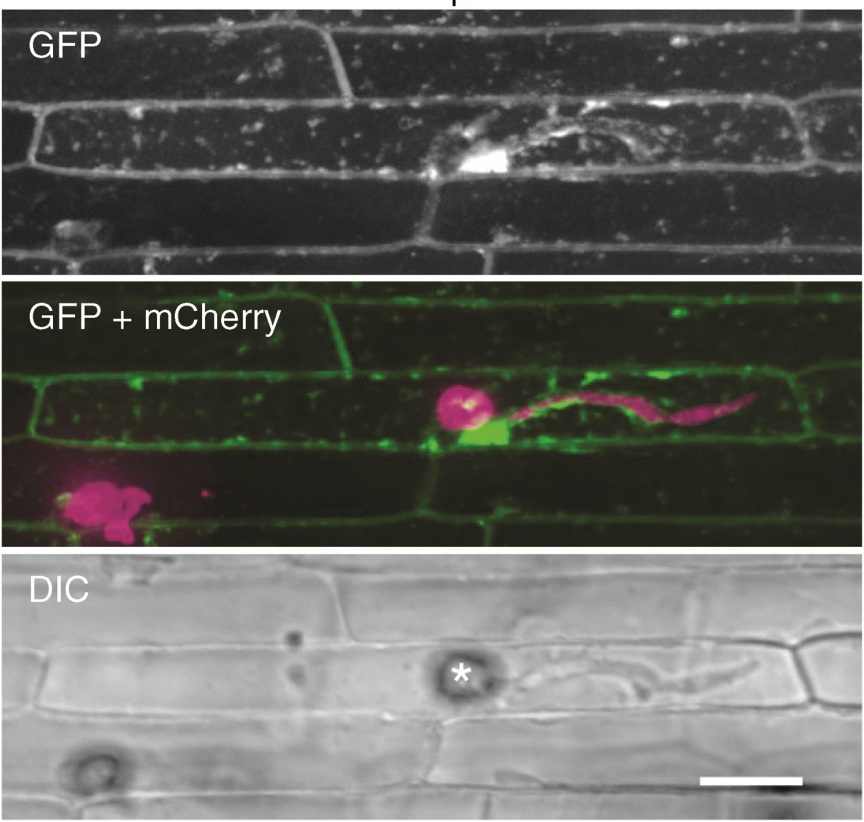

$42 \mathrm{hpi}$
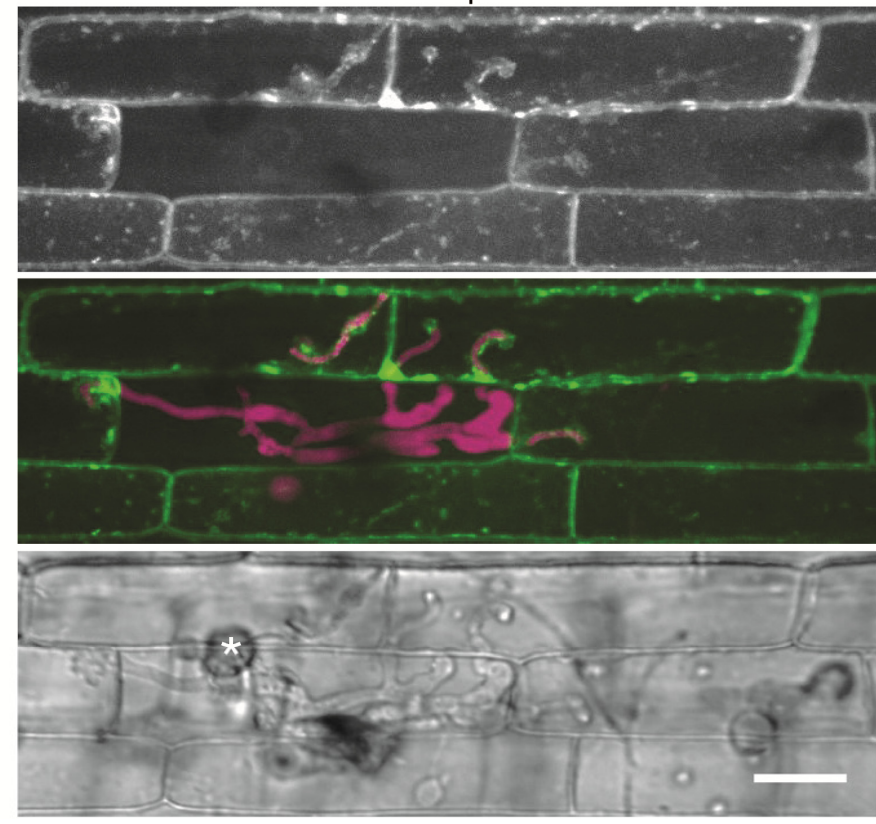

Fig. 4. Subcellular localization of OsCERK1-GFP (green fluorescent protein). Rice leaf sheaths of transgenic plants expressing OsCERK1-GFP were inoculated with conidial suspension of Magnaporthe oryzae expressing mCherry. GFP = Stacked Z-series confocal GFP fluorescence images, GFP+mCherry = superimposed images of stacked Z-series confocal fluorescence images of GFP (green) and mCherry (magenta), DIC = corresponding differential interference contrast images. Asterisks indicate positions of appressoria (i.e., penetration sites). Bars $=20 \mu \mathrm{m}$. 
We also showed that OsCERK1 contributes to immunity against $M$. oryzae. We recently reported a slight but significant reduction in immunity to this fungus in $C E B i P$-disrupted plants (Kouzai et al. 2014). OsCERK1-disrupted plants also showed a significant reduction in the early infection stages, suggesting that the perception of $\mathrm{CE}$ by the host is a substantial obstacle for the rice blast fungus. The significance of $\mathrm{CE}$ perception in rice immunity is also supported by studies on $M$. oryzae. Infection hyphae of $M$. oryzae in the first-invaded rice cell showed only weak staining with a fluorescent probe to detect chitin (Mochizuki et al. 2011), suggesting the presence of a fungal strategy to minimize the detection of cell wall chitin by the host plant during invasion. In fact, $\alpha-1,3$-glucan that masks the chitin layer in fungal cell walls was shown to be indispensable for successful fungal invasion (Fujikawa et al. 2012). In addition, $M$. oryzae secretes an effector protein, secreted LysM protein 1 (Slp1), to capture CE in competition with CEBiP for full virulence (Mentlak et al. 2012). Therefore, suppression of rice immunity induced by $\mathrm{CE}$ is an important stealth mechanism of M. oryzae for successful infection. LYP4 and LYP6, CEBiP-like proteins in rice, were reported to participate in rice immunity to M. oryzae and bind directly with chitin (Liu et al. 2012), although we did not detect any interaction other than CEBiP in affinity-labeling analysis using biotinylated CE (Kouzai et al. 2014). Given that this inconsistency in results was not because of differences in experimental conditions, either LYP4, LYP6, or both might play a role in immunity to $M$. oryzae through an asyet-unknown mechanism other than $\mathrm{CE}$ recognition.

Considerable progress has been made in understanding signal transduction through PRR (Kawano and Shimamoto 2013; Monaghan and Zipfel 2012). However, little is known about the dynamic behavior of PRR during the host-pathogen interaction. Transient expression of OsCERK1-GFP in rice protoplasts showed that OsCERK1 was transported from the endoplasmic reticulum to the PM by vesicle trafficking (Chen et al. 2010). Consistent with the observation, OsCERK1-GFP was detected
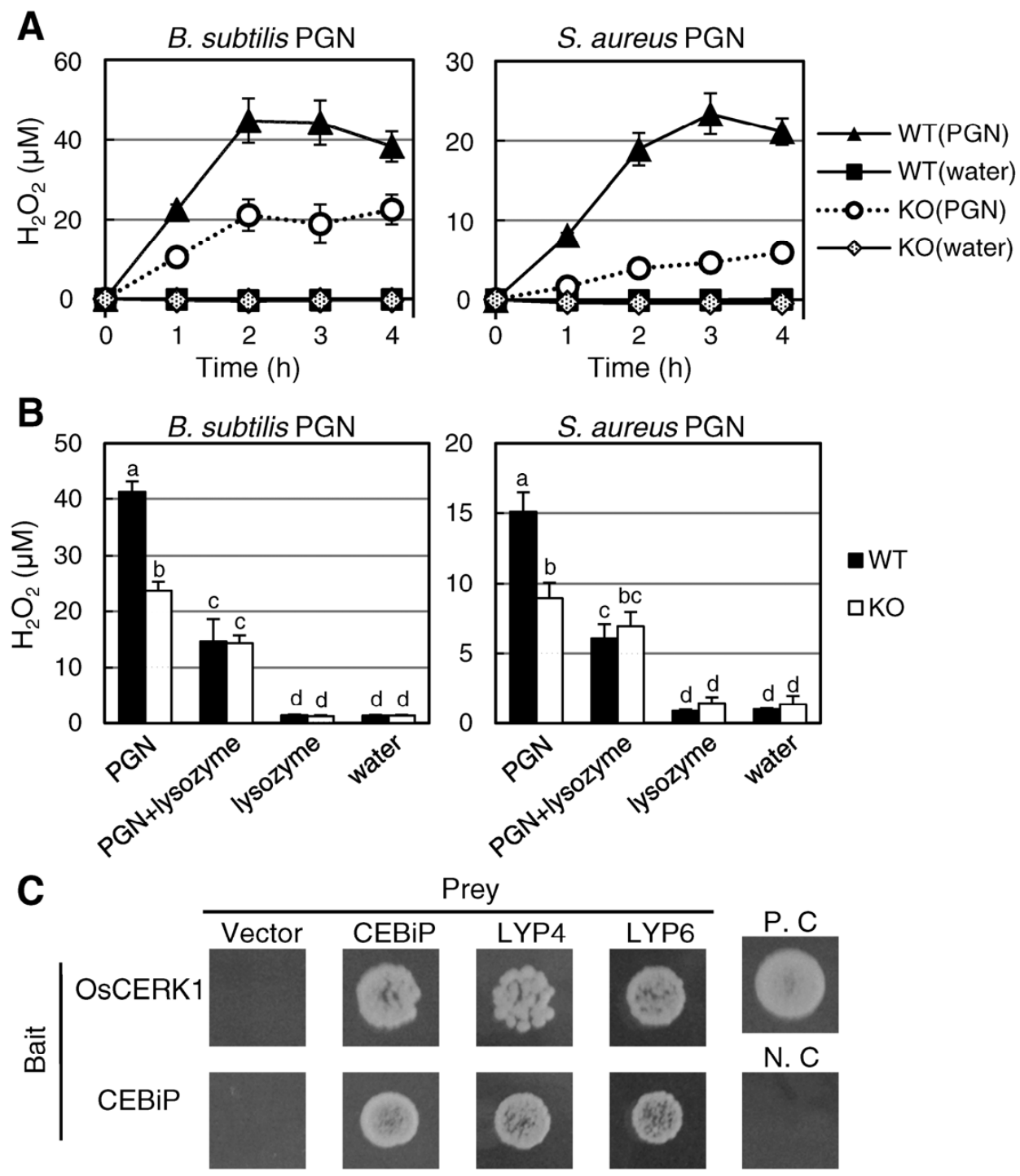

Fig. 5. Responses to peptidoglycan (PGN). A, Generation of $\mathrm{H}_{2} \mathrm{O}_{2}$ in response to $\mathrm{PGN}$ in an oscerk1 line (line 53; KO) and nontransformants (WT). Suspension-cultured cells were treated with PGN at $10 \mu \mathrm{g} \mathrm{ml}^{-1}$ derived from Bacillus subtilis or Staphylococcus aureus, and $\mathrm{H}_{2} \mathrm{O}_{2}$ in the culture medium was quantified. Error bars represent standard deviation ( $n=3$ independent treatments). B, Amount of $\mathrm{H}_{2} \mathrm{O}_{2}$ after treatment with lysozyme-treated PGN in an oscerk1 line (line 53; KO) and nontransformants (WT). Suspension-cultured cells were treated with PGN at $10 \mu \mathrm{g} \mathrm{ml}^{-1}$, lysozyme-pretreated PGN (containing lysozyme at $\left.1 \mu \mathrm{g} \mathrm{ml}^{-1}\right)$ at $10 \mu \mathrm{g} \mathrm{ml}^{-1}$, or lysozyme at $1 \mu \mathrm{g} \mathrm{ml}^{-1} \cdot \mathrm{H}_{2} \mathrm{O}_{2}$ in the culture medium was quantified at $2 \mathrm{~h}$. Error bars represent standard deviation $(n=3$ independent treatments). Different letters above bars indicate significant differences at $P<0.05$ (Tukey's test). C, Interactions between rice LysM-containing receptors. Yeast two-hybrid assay was performed using extracellular domains of OsCERK1 and CEBiP as bait and three CEBiP-type receptors (CEBiP, LYP4, and LYP6) as prey. Growth on selective medium (SD/-LWHA) indicated positive interaction. Vector indicates empty vector (control). P.C. = positive control using pGBKT7-53 and pGADT7-T vectors and N.C. = negative control using pGBKT7-Lam and pGADT7-T vectors. 
in the PM, the endoplasmic reticulum-like structures, and vesicle-like organelles in leaf sheath cells of stable transformants. In the inoculated cells, OsCERK1-GFP was localized around the primary infection hyphae in the first- and second-invaded rice cells. This observation is consistent with previous reports that primary infection hyphae of $M$. oryzae invaginate the host PM (Koga 1994) and that infection hyphae of $M$. oryzae are encased by a membrane known as the extra-invasive hyphal membrane (EIHM) (Kankanala et al. 2007), which originates from the rice PM (Mentlak et al. 2012). However, OsCERK1-GFP was not detected as encasing the whole invasive hyphae, which was inconsistent with the result using transgenic rice expressing an artificial PM marker gene, LTi6B-GFP (Mentlak et al. 2012). Our observations suggest that $\mathrm{CE}$ perception around the infection hyphae might be limited to the early stages, when infection hyphae have just invaded rice cells, and also imply that the EIHM differs from the authentic PM, at least with regard to resident proteins. Similar results have been reported for the extrahaustorial membrane of powdery mildews and oomycetes (Koh et al. 2005; Lu et al. 2012).

In Arabidopsis, AtCERK1 also mediates the PGN response but needs the CEBiP-like proteins LYM1 and LYM3, which directly bind with PGN (Willmann et al. 2011). The rice homologs of LYM1 or LYM3, LYP4 and LYP6, also participate in the PGN response (Liu et al. 2012). Our data demonstrate that rice cells respond to two different types of PGN: a meso-diaminopimelate-type from B. subtilis and an L-lysine-type from $S$. aureus. In addition, our results indicate that OsCERK1 is involved in the perception of both types of PGN, probably by interacting with one or both LYP4 and LYP6. However, PGN
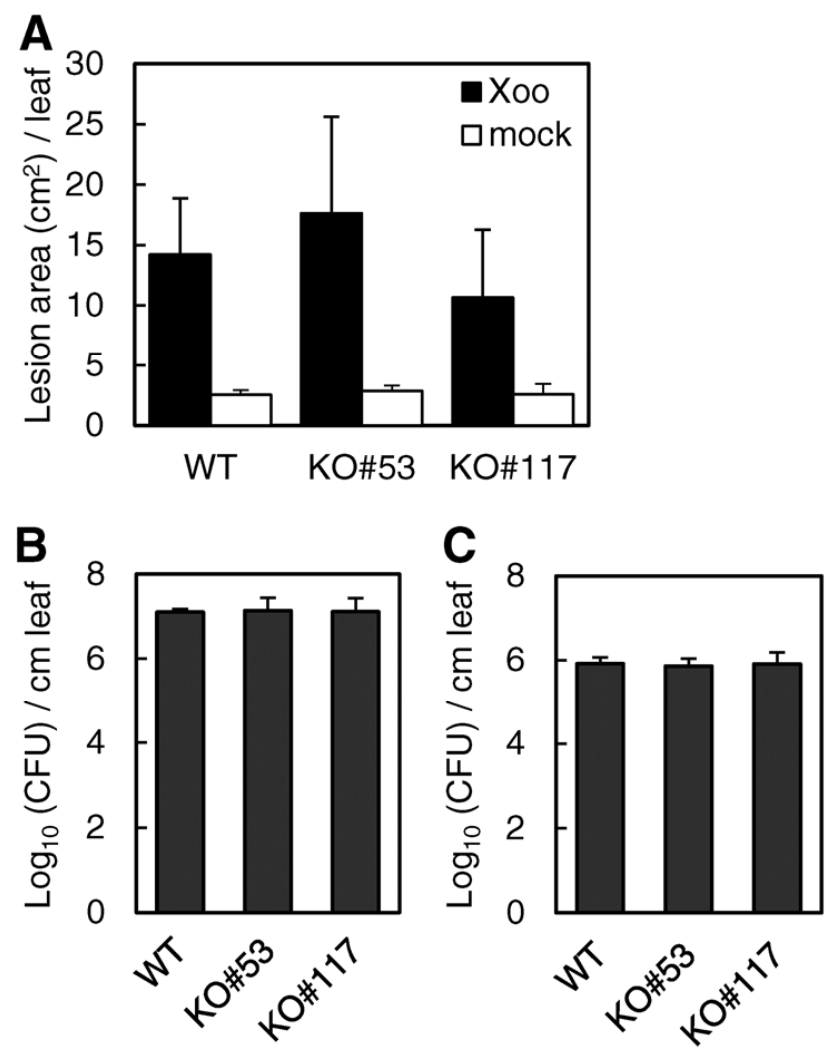

Fig. 6. Effect of OsCERK1 disruption on immunity to Xanthomonas oryzae pv. oryzae (Xoo). A, Lesion area at 14 days postinoculation with $X$. oryzae pv. oryzae. B, Bacterial population at 4 days postinoculation with $X$. oryzae pv. oryzae. C, Bacterial population at 4 days postinoculation with $X$. oryzae pv. oryzae $h r p X$ mutant. Fully expanded upper leaves of glasshouse-grown plants were inoculated by the clipping method. Data are means \pm standard deviation $(n=4$ plants $)$. responsiveness in oscerkl cells was not completely abolished, and pretreatment of PGN with lysozyme tended to lower the responsiveness of the oscerkl lines. These results imply that other RLK are also involved in perceiving the glycan chains in PGN. The residual responsiveness to lysozyme-treated PGN in both the wild-type and oscerkl lines raises the possibility that rice has another one or more receptors for the lysozyme-resistant structure in PGN, or that the PGN preparations used in these experiments were contaminated with another one or more elicitors. Further studies are needed to elucidate the molecular mechanism underlying the $\mathrm{PGN}$ recognition in rice.

In the experimental conditions we used, oscerkl did not show impaired immunity to $X$. oryzae pv. oryzae. This result might be partly ascribed to redundancy among RLK in PGN perception, and also to other perception mechanisms for bacterial MAMPs, including flg22 in rice. Moreover, Yamaguchi and associates (2013) recently reported that Xoo1488, an effector of $X$. oryzae pv. oryzae, suppressed the OsCERK1-mediated phosphorylation of a receptor-like cytoplasmic kinase, OsRLCK185, and that overexpression of Xool488 in rice reduced the immunity to $X$. oryzae pv. oryzae. The existence of suppression mechanisms targeting the signaling pathways downstream of OsCERK1 might also be a reason why altered immunity to $X$. oryzae pv. oryzae was not detected in oscerk1. In contrast, reduced expression of $L Y P 4$ or $L Y P 6$ led to impaired immunity to $X$. oryzae pv. oryzae (Liu et al. 2012). Given that this inconsistency in results was not caused by differences in experimental conditions, we presume that either LYP4 or LYP6, or both, play other roles besides PGN recognition.

Taken together, our data indicate that OsCERK1 mediates the perception of both fungal and bacterial MAMPs by interacting with different LysM-RLP. The present study also demonstrates the usefulness of a gene-disrupted mutant. The roles of LysM-RLK are an emerging subject in both plant immunity and symbioses (Gust et al. 2012; Nakagawa et al. 2011); therefore, OsCERK1-disrupted plants will be useful materials to further investigate plant-microbe interactions.

\section{MATERIALS AND METHODS}

\section{Plant and fungus materials and PCR primers.}

We used rice (Oryza sativa L. japonica 'Nipponbare Kanto BL number 2') harboring Pii and Pish, resistance genes against the pathogen $M$. oryzae. Rice plants were grown hydroponically in nutrient solution under a photoperiod of $14 \mathrm{~h}$ of light $\left(28^{\circ} \mathrm{C}\right)$ and $10 \mathrm{~h}$ of darkness $\left(25^{\circ} \mathrm{C}\right)$. Rice calli were cultivated on N6D plates (Toki et al. 2006) containing 0.4\% (wt/vol) Gelrite and hygromycin at $50 \mu \mathrm{g} \mathrm{ml}^{-1}$ and $28^{\circ} \mathrm{C}$. The $M$. oryzae strains Guy11 (race 134.1, avrPish; provided by M. Nishimura) (Leung et al. 1988) and Ina86-137 (race 007.0; MAFF101511) were cultured on oatmeal agar plates at $26^{\circ} \mathrm{C}$ to induce conidiation. Sequences of all primers used in this study are listed in Supplementary Table S4.

\section{Gene targeting.}

Vector construction and rice transformation for gene targeting were performed as described by Ozawa and associates (2012). A 6.4-kb fragment corresponding to the upper region of the $O s C E R K 1$ promoter and a 5.6-kb fragment corresponding to the lower region from the first exon of OsCERK1 were PCR-amplified from rice total DNA. Both fragments were cloned into an entry vector containing the HPT cassette. The resultant vector and two other entry vectors containing the $D T$ $A$ gene cassette were combined with the destination binary vector using the Gateway system (Invitrogen, Carlsbad, CA, U.S.A.). The T-DNA region of the resultant binary vector is shown in Figure 1A. 
For complementation experiments, the OsCERK1 cDNA under the control of the rice actin promoter in the binary vector pSTARA R-5 (Inplanta Innovations, Yokohama, Japan) was introduced into oscerkl calli derived from $\mathrm{T}_{2}$ seed of line number 53. The transcript levels of $O s C E R K 1$ in bispyribac-resistant calli were analyzed by quantitative reverse-transcriptase (RT)PCR. We used two lines with OsCERK1 transcript levels similar to that in nontransformed calli for complementation tests.

\section{Screening of targeted calli and genotyping.}

Genomic DNA was extracted from calli grown on selection medium containing hygromycin at $50 \mathrm{mg} \mathrm{liter}^{-1}$ and subjected to PCR using KOD FX Neo polymerase (Toyobo, Osaka, Japan). We regenerated gene-targeted calli and obtained selfpollinated seed $\left(\mathrm{T}_{1}\right.$ generation). Genotyping of the OsCERK1 locus of $\mathrm{T}_{1}$ seedlings and calli induced from $\mathrm{T}_{1}$ seed was performed by PCR.

\section{RT-PCR.}

Transcriptional expression was confirmed by RT-PCR using EX Taq (Takara, Ohtsu, Japan). Quantitative RT-PCR was performed using $1 \times$ SYBR Premix Ex Taq II (Takara). The relative levels of gene expression were quantified using MX3000P (Stratagene, La Jolla, CA, U.S.A.). The data were normalized to those of the elongation factor gene $e E F-1 \alpha$ (Jain et al. 2006).

\section{Measurement of $\mathrm{H}_{2} \mathrm{O}_{2}$ generation.}

Suspension-cultured cells $(100 \mathrm{mg})$ were treated with $\mathrm{N}$-acetylchitoheptaose at $100 \mathrm{ng} \mathrm{ml}^{-1}$, PGN from B. subtilis or $S$. aureus (Sigma-Aldrich, St. Louis) at $10 \mu \mathrm{g} \mathrm{ml}^{-1}$, or $100 \mathrm{nM}$ flg22 at $28^{\circ} \mathrm{C}$ with shaking at $800 \mathrm{rpm}$. PGN $\left(1 \mathrm{mg} \mathrm{ml}^{-1}\right)$ was digested with chicken egg-white lysozyme (Sigma-Aldrich) at $50 \mu \mathrm{g} \mathrm{ml}^{-1}$ for $16 \mathrm{~h}$ at $37^{\circ} \mathrm{C} . \mathrm{H}_{2} \mathrm{O}_{2}$ in the culture medium was quantified by the luminol-dependent chemiluminescence assay, as described by Kouzai and associates (2013).

\section{Microarray analysis.}

Total RNA was extracted from suspension-cultured cells treated with $N$-acetylchitoheptaose at $100 \mathrm{ng} \mathrm{ml}^{-1}$ for $1 \mathrm{~h}$. Mock treatment was used as the control. All samples were prepared in triplicate. Microarray analyses were performed using rice oligo microarrays with 44k features (Agilent Technologies, Palo Alto, CA, U.S.A.), as described by Takehisa and associates (2012). Data were analyzed using the Subio Platform.

\section{Disease resistance test.}

The degree of hyphal growth in leaf sheath epidermal cells was analyzed as described by Tanabe and associates (2009). Briefly, leaf sheaths from the sixth leaves were excised, the inside filled with a conidial suspension $\left(1 \times 10^{5}\right.$ conidia $\left.\mathrm{ml}^{-1}\right)$, and incubated for $48 \mathrm{~h}$ at $25^{\circ} \mathrm{C}$ in the dark. To evaluate immunity to $X$. oryzae pv. oryzae, fully expanded upper leaves of glasshouse-grown plants were inoculated by clipping leaf tips with scissors that had been immersed in a bacterial suspension (optical density at $600 \mathrm{~nm}=0.3$ ). To quantify the bacterial population, the inoculated leaves $(1 \mathrm{~cm}$ long) were ground in and diluted with sterile water, then plated on nutrient broth yeast extract medium. After incubation for 2 days at $28^{\circ} \mathrm{C}$, the colony numbers were counted.

\section{Preparation of rice and $M$. oryzae transformants for fluorescence imaging.}

The coding region of OsCERK1 was cloned between the Cauliflower mosaic virus $35 \mathrm{~S}$ promoter and GFP in the binary vector pGWB5 (Nakagawa et al. 2007), which was introduced into rice plants by Agrobacterium-mediated transformation (Toki et al. 2006). The promoter region of the translation elon- gation factor gene of Aureobasidium pullulans (Vanden Wymelenberg et al. 1997) fused to mCherry (Clontech, Palo Alto, CA, U.S.A.) was cloned in a binary vector to construct pCAMBIA-TCT, which was introduced into M. oryzae Ina86137 via Agrobacterium-mediated transformation (Saitoh et al. 2008).

\section{Confocal microscopy.}

OsCERK1-GFP and mCherry fluorescence were observed under a fluorescence microscope (DM6000B; Leica Microsystems, Wetzlar, Germany) equipped with a confocal laser-scanning unit (CSU-X1; Yokogawa Electric, Tokyo), laser units (Sapphire 488 and $561 \mathrm{~nm}$; Coherent Inc., Santa Clara, CA, U.S.A.), a dichroic mirror (DM-405/488/561), and emission filters (GFP, EM-520/35; mCherry, EM617/73). Fluorescence images were collected with an EM-CCD camera (iXon897; Andor Technology, South Windsor, CT, U.S.A.) using a $\times 40$ objective and were analyzed with MetaMorph software (Molecular Devices, Sunnyvale, CA, U.S.A.).

\section{Yeast two-hybrid assay.}

A yeast two-hybrid assay was performed using the MATCHMAKER GAL4 Two-Hybrid System 3 according to the manufacturer's protocol (Clontech). The extracellular regions of OsCERK1, CEBiP, LYP4, and LYP6 were amplified by PCR and cloned into pGBKT7 or pGADT7, as described by Shimizu and associates (2010).

\section{ACKNOWLEDGMENTS}

We thank F. Takaiwa for providing plasmid vectors used for gene targeting, H. Ochiai for providing the $X$. oryzae pv. oryzae hrpX mutant, $\mathrm{Y}$. Nagamura and R. Motoyama for their support in the microarray analyses, K. Saitoh for constructing pCAMBIA-TCT, E. Nakajima for rice transformation, N. Aoyagi for X. oryzae pv. oryzae resistance tests, and Y. Ninomiya and $\mathrm{H}$. Sekou of Meiji University for the help with the $\mathrm{H}_{2} \mathrm{O}_{2}$ assay. This work was supported by grants from the Japan Society for the Promotion of Science initiated by the Council for Science and Technology Policy (GS028 to Y. Nishizawa) and from the Ministry of Education, Culture, Sports, Science and Technology, Japan (number 22248041 to $\mathrm{N}$. Shibuya and number 22570052 to H. Kaku).

\section{LITERATURE CITED}

Boller, T., and Felix, G. 2009. A renaissance of elicitors: perception of microbe-associated molecular patterns and danger signals by patternrecognition receptors. Annu. Rev. Plant Biol. 60:379-406.

Chen, L., Hamada, S., Fujiwara, M., Zhu, T., Thao, N. P., Wong, H. L. Krishna, P., Ueda, T., Kaku, H., Shibuya, N., Kawasaki, T., and Shimamoto, K. 2010. The Hop/Sti1-Hsp90 chaperone complex facilitates the maturation and transport of a PAMP receptor in rice innate immunity. Cell Host Microbe 7:185-196.

Erbs, G., Silipo, A., Aslam, S., De Castro, C., Liparoti, V., Flagiello, A. Pucci, P., Lanzetta, R., Parrilli, M., Molinaro, A., Newman, M. A., and Cooper, R. M. 2008. Peptidoglycan and muropeptides from pathogens Agrobacterium and Xanthomonas elicit plant innate immunity: Structure and activity. Chem. Biol. 15:438-448.

Fujikawa, T., Sakaguchi, A., Nishizawa, Y., Kouzai, Y., Minami, E., Yano, S., Koga, H., Meshi, T., and Nishimura, M. 2012. Surface $\alpha$-1,3-glucan facilitates fungal stealth infection by interfering with innate immunity in plants. PLoS Pathog. 8:e1002882.

Gomez-Gomez, L., and Boller, T. 2000. FLS2: An LRR receptor-like kinase involved in the perception of the bacterial elicitor flagellin in Arabidopsis. Mol. Cell 5:1003-1011.

Gust, A. A., Biswas, R., Lenz, H. D., Rauhut, T., Ranf, S., Kemmerling, B., Gotz, F., Glawischnig, E., Lee, J., Felix, G., and Nurnberger, T. 2007. Bacteria-derived peptidoglycans constitute pathogen-associated molecular patterns triggering innate immunity in Arabidopsis. J. Biol. Chem. 282:32338-32348.

Gust, A. A., Willmann, R., Desaki, Y., Grabherr, H. M., and Nurnberger, T. 2012. Plant LysM proteins: Modules mediating symbiosis and immunity. Trends Plant Sci. 17:495-502.

Iizasa, E., Mitsutomi, M., and Nagano, Y. 2010. Direct binding of a plant 
LysM receptor-like kinase, LysM RLK1/CERK1, to chitin in vitro. J. Biol. Chem. 285:2996-3004.

Jain, M., Nijhawan, A., Tyagi, A. K., and Khurana, J. P. 2006. Validation of housekeeping genes as internal control for studying gene expression in rice by quantitative real-time PCR. Biochem. Biophys. Res. Commun. 345:646-651.

Jasin, M., Moynahan, M. E., and Richardson, C. 1996. Targeted transgenesis. Proc. Natl. Acad. Sci. U.S.A. 93:8804-8808

Jeon, J. S., Lee, S., Jung, K. H., Jun, S. H., Jeong, D. H., Lee, J., Kim, C., Jang, S., Yang, K., Nam, J., An, K., Han, M. J., Sung, R. J., Choi, H. S., Yu, J. H., Choi, J. H., Cho, S. Y., Cha, S. S., Kim, S. I., and An, G. 2000. T-DNA insertional mutagenesis for functional genomics in rice. Plant J. 22:561-570.

Kaku, H., Nishizawa, Y., Ishii-Minami, N., Akimoto-Tomiyama, C., Dohmae, N., Takio, K., Minami, E., and Shibuya, N. 2006. Plant cells recognize chitin fragments for defense signaling through a plasma membrane receptor. Proc. Natl. Acad. Sci. U.S.A. 103:11086-11091.

Kankanala, P., Czymmek, K., and Valent, B. 2007. Roles for rice membrane dynamics and plasmodesmata during biotrophic invasion by the blast fungus. Plant Cell 19:706-724.

Kawano, Y., and Shimamoto, K. 2013. Early signaling network in rice PRR-mediated and R-mediated immunity. Curr. Opin. Plant Biol. 16:496-504

Kishimoto, K., Kouzai, Y., Kaku, H., Shibuya, N., Minami, E., and Nishizawa, Y. 2010. Perception of the chitin oligosaccharides contributes to disease resistance to blast fungus Magnaporthe oryzae in rice. Plant J. 64:343354

Koga, H. 1994. Hypersensitive death, autofluorescence, and ultrastructural changes in cells of leaf sheaths of susceptible and resistant near-isogenic lines of rice $(P i-z t)$ in relation of penetration and growth of Pyricularia oryzae. Can. J. Bot. 72:1463-1477.

Koh, S., Andre, A., Edwards, H., Ehrhardt, D., and Somerville, S. 2005 Arabidopsis thaliana subcellular responses to compatible Erysiphe cichoracearum infections. Plant J. 44:516-529.

Kombrink, A., Sanchez-Vallet, A., and Thomma, B. P. 2011. The role of chitin detection in plant-pathogen interactions. Microbes Infect. 13:11681176.

Kouzai, Y., Kaku, H., Shibuya, N., Minami, E., and Nishizawa, Y. 2013. Expression of the chimeric receptor between the chitin elicitor receptor $\mathrm{CEBiP}$ and the receptor-like protein kinase Pi-d2 leads to enhanced responses to the chitin elicitor and disease resistance against Magnaporthe oryzae in rice. Plant Mol. Biol. 81:287-295.

Kouzai, Y., Nakajima, K., Hayafune, M., Ozawa, K., Kaku, H., Shibuya, N., Minami, E., and Nishizawa, Y. 2014. CEBiP is the major chitin oligomer-binding protein in rice and plays a main role in the perception of chitin oligomers. Plant Mol. Biol. 84:519-528.

Leung, H., Borromoeo, E. S., Bernardo, M. A., and Notteghem, J. L. 1988 Genetic analysis of virulence in the rice blast fungus Magnaporthe grisea. Phytopathology 78:1227-1233.

Liu, B., Li, J. F., Ao, Y., Qu, J., Li, Z., Su, J., Zhang, Y., Liu, J., Feng, D., Qi, K., He, Y., Wang, J., and Wang, H. B. 2012. Lysin motif-containing proteins LYP4 and LYP6 play dual roles in peptidoglycan and chitin perception in rice innate immunity. Plant Cell 24:3406-3419.

Lu, Y. J., Schornack, S., Spallek, T., Geldner, N., Chory, J., Schellmann, S., Schumacher, K., Kamoun, S., and Robatzek, S. 2012. Patterns of plant subcellular responses to successful oomycete infections reveal differences in host cell reprogramming and endocytic trafficking. Cell Microbiol. 14:682-697.

Mentlak, T. A., Kombrink, A., Shinya, T., Ryder, L. S., Otomo, I., Saitoh, H., Terauchi, R., Nishizawa, Y., Shibuya, N., Thomma, B. P., and Talbot, N. J. 2012. Effector-mediated suppression of chitin-triggered immunity by Magnaporthe oryzae is necessary for rice blast disease. Plant Cell 24:322-335

Miya, A., Albert, P., Shinya, T., Desaki, Y., Ichimura, K., Shirasu, K., Narusaka, Y., Kawakami, N., Kaku, H., and Shibuya, N. 2007. CERK1, a LysM receptor kinase, is essential for chitin elicitor signaling in Arabidopsis. Proc. Natl. Acad. Sci. U.S.A. 104:19613-19618.

Miyao, A., Tanaka, K., Murata, K., Sawaki, H., Takeda, S., Abe, K., Shinozuka, Y., Onosato, K., and Hirochika, H. 2003. Target site specificity of the Tos 17 retrotransposon shows a preference for insertion within genes and against insertion in retrotransposon-rich regions of the genome. Plant Cell 15:1771-1780.

Mochizuki, S., Saitoh, K., Minami, E., and Nishizawa, Y. 2011. Localization of probe-accessible chitin and characterization of genes encoding chitin-binding domains during rice-Magnaporthe oryzae interactions. J. Gen. Plant Pathol. 77:163-173.

Monaghan, J., and Zipfel, C. 2012. Plant pattern recognition receptor complexes at the plasma membrane. Curr. Opin. Plant Biol. 15:349-357.
Nakagawa, T., Kurose, T., Hino, T., Tanaka, K., Kawamukai, M., Niwa, Y, Toyooka, K., Matsuoka, K., Jinbo, T., and Kimura, T. 2007. Development of series of gateway binary vectors, pGWBs, for realizing efficient construction of fusion genes for plant transformation. J. Biosci. Bioeng. 104:34-41.

Nakagawa, T., Kaku, H., Shimoda, Y., Sugiyama, A., Shimamura, M., Takanashi, K., Yazaki, K., Aoki, T., Shibuya, N., and Kouchi, H. 2011. From defense to symbiosis: Limited alterations in the kinase domain of LysM receptor-like kinases are crucial for evolution of legume-Rhizobium symbiosis. Plant J. 65:169-180.

Ozawa, K., Wakasa, Y., Ogo, Y., Matsuo, K., Kawahigashi, H., and Takaiwa, F. 2012. Development of an efficient Agrobacterium-mediated gene targeting system for rice and analysis of rice knockouts lacking granule-bound starch synthase (Waxy) and $\beta 1,2$-xylosyltransferase. Plant Cell Physiol. 53:755-761.

Petutschnig, E. K., Jones, A. M., Serazetdinova, L., Lipka, U., and Lipka, V. 2010. The lysin motif receptor-like kinase (LysM-RLK) CERK1 is a major chitin-binding protein in Arabidopsis thaliana and subject to chitin-induced phosphorylation. J. Biol. Chem. 285:28902-28911.

Robatzek, S., Chinchilla, D., and Boller, T. 2006. Ligand-induced endocytosis of the pattern recognition receptor FLS2 in Arabidopsis. Genes Dev. 20:537-542.

Saitoh, K., Nishimura, M., Kubo, Y., Hayashi, N., Minami, E., and Nishizawa, Y. 2008. Construction of a binary vector for knockout and expression analysis of rice blast fungus genes. Biosci. Biotechnol. Biochem. 72:1380-1383.

Shimizu, T., Nakano, T., Takamizawa, D., Desaki, Y., Ishii-Minami, N., Nishizawa, Y., Minami, E., Okada, K., Yamane, H., Kaku, H., and Shibuya, N. 2010. Two LysM receptor molecules, CEBiP and OsCERK1, cooperatively regulate chitin elicitor signaling in rice. Plant J. 64:204-214.

Shinya, T., Motoyama, N., Ikeda, A., Wada, M., Kamiya, K., Hayafune, M., Kaku, H., and Shibuya, N. 2012. Functional characterization of CEBiP and CERK1 homologs in Arabidopsis and rice reveals the presence of different chitin receptor systems in plants. Plant Cell Physiol. 53:1696-1706.

Takehisa, H., Sato, Y., Igarashi, M., Abiko, T., Antonio, B.A., Kamatsuki, K., Minami, H., Namiki, N., Inukai, Y., Nakazono, M., and Nagamura, Y. 2012. Genome-wide transcriptome dissection of the rice root system: Implications for developmental and physiological functions. Plant J. 69:126-140.

Tanabe, S., Nishizawa, Y., and Minami, E. 2009. Effects of catalase on the accumulation of $\mathrm{H}_{2} \mathrm{O}_{2}$ in rice cells inoculated with rice blast fungus, Magnaporthe oryzae. Physiol. Plant. 137:148-154.

Terada, R., Urawa, H., Inagaki, Y., Tsugane, K., and Iida, S. 2002. Efficient gene targeting by homologous recombination in rice. Nat. Biotechnol. 20:1030-1034

Toki, S., Hara, N., Ono, K., Onodera, H., Tagiri, A., Oka, S., and Tanaka, H. 2006. Early infection of scutellum tissue with Agrobacterium allows high-speed transformation of rice. Plant J. 47:969-976.

Vanden Wymelenberg, A. J., Cullen, D., Spear, R. N., Schoenike, B., and Andrews, J. H. 1997. Expression of green fluorescent protein in Aureobasidium pullulans and quantification of the fungus on leaf surfaces. BioTechniques 23:686-690.

Wan, J., Zhang, X. C., Neece, D., Ramonell, K. M., Clough, S., Kim, S. Y., Stacey, M. G., and Stacey, G. 2008. A LysM receptor-like kinase plays a critical role in chitin signaling and fungal resistance in Arabidopsis. Plant Cell 20:471-481.

Willmann, R., Lajunen, H. M., Erbs, G., Newman, M. A., Kolb, D., Tsuda, K., Katagiri, F., Fliegmann, J., Bono, J. J., Cullimore, J. V., Jehle, A. K. Gotz, F., Kulik, A., Molinaro, A., Lipka, V., Gust, A. A., and Nurnberger, T. 2011. Arabidopsis lysin-motif proteins LYM1 LYM3 CERK1 mediate bacterial peptidoglycan sensing and immunity to bacterial infection. Proc. Natl. Acad. Sci. U.S.A. 108:19824-19829.

Yamaguchi, K., Yamada, K., Ishikawa, K., Yoshimura, S., Hayashi, N., Uchihashi, K., Ishihama, N., Kishi-Kaboshi, M., Takahashi, A., Tsuge, S., Ochiai, H., Tada, Y., Shimamoto, K., Yoshioka, H., and Kawasaki, T. 2013. A receptor-like cytoplasmic kinase targeted by a plant pathogen effector is directly phosphorylated by the chitin receptor and mediates rice immunity. Cell Host Microbe 13:347-357.

Zipfel, C., Kunze, G., Chinchilla, D., Caniard, A., Jones, J. D., Boller, T., and Felix, G. 2006. Perception of the bacterial PAMP EF-Tu by the receptor EFR restricts Agrobacterium-mediated transformation. Cell 125:749-760.

\section{AUTHOR-RECOMMENDED INTERNET RESOURCE}

Subio Inc. website: www.subio.jp 